\title{
Dermatitis artefacta as a symptom of schizophrenia?
}

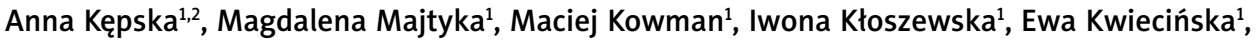 \\ Anna Zalewska-Janowska²
}

\author{
${ }^{1}$ Department of Old Age Psychiatry and Psychotic Disorders, Medical University of Lodz, Poland \\ Head of Department: Prof. Iwona Kłoszewska MD, PhD \\ ${ }^{2}$ Psychodermatology Department, Medical University of Lodz, Poland \\ Head of Department: Prof. Anna Zalewska-Janowska MD, PhD
}

Postep Derm Alergol 2014; XXXI, 4: 277-279

DOI: $10.5114 /$ pdia.2014.40921

\begin{abstract}
Dermatitis artefacta is a disease that occurs as a result of a self-inflicted injury of the skin. The skin lesions are most often located on the areas within easy reach of the patient's dominant hand sparing the middle part of the back. Dermatitis artefacta may coexist with psychiatric disorders and imitate many dermatologic diseases. As most of the patients with self-inflicted dermatoses usually initially deny any psychiatric problems, what delays psychiatric intervention, they are typically first seen by dermatologists. We are reporting a case of a 35-year-old man with a 3-year-long history of schizophrenia who has been treated at a dermatologist's office sequentially with acne, bacterial lesions, suspected tuberculosis. However, the treatment was ineffective. He was diagnosed with dermatitis artefacta after 7 years of disease duration. During this time he was treated with many medicines e.g. isotretinoin, which is contraindicated in psychosis as it worsens the course of disease. After establishing the correct diagnosis and antipsychotic treatment, a significant improvement was obtained in both skin condition and mental state. These are the reasons why we would like to recommend close cooperation between dermatologists and psychiatrists.
\end{abstract}

Key words: dermatitis artefacta, schizophrenia, self-mutilation, psychodermatology.

\section{Introduction}

Lack of insight is common in many psychiatric patients, especially suffering from psychotic disorders. Patients may not believe that they are sick or abnormal in any way, and because of a high level of stigmatization, a decision to undergo psychiatric treatment is difficult. That results in diagnosis of medical diseases other than psychiatric ones. The incidence of dermatitis artefacta among dermatologic patients has been reported to be about $0.3 \%$ and treatment usually starts at the dermatologist's office [1]. The aim of this paper is to alert the clinicians that different psychiatric disorders may manifest as dermatitis artefacta and to provide tips to dermatologists helping them to diagnose a true psychiatric disease.

\section{Case report}

A 35-year-old married male with a 3-year history of schizophrenia was referred to the psychiatric clinic. He had already been treated by a dermatologist as a case of severe acne for many years with no effect. On admission our patient confirmed psychotic symptoms such as: thought insertion, passivity, somatic hallucinations, persecutory and hypochondriac delusions, as well as delusions of jealousy. Disorganization of thinking, bizarre behavior and a high level of anxiety was noticed. He had no insight into his condition. The patient's delusional activity seriously affected his social and family life. There were no abnormalities in general physical examination and laboratory tests were within normal limits.

The patient's face and thighs were covered with multiple itchy skin lesions. He reported that these lesions had appeared 12 years earlier and had been caused by a car accident with head injury and also some marital problems. The patient had a long history of dermatological treatment (e.g. with isotretinoin) which started 7 years earlier. On his first visit in the dermatological out-patient clinic he presented both primary and secondary skin lesions: pustules, atrophic and hypertrophic scars and a painless ulcer of $3 \mathrm{~cm}$ in diameter on his chin. Most of lesions had geometrical shape. Histopathological exam-

Address for correspondence: Anna Kępska MD, Psychodermatology Department, Medical University of Lodz, 251 Pomorska St, 92-213 Lodz, Poland, phone: +48 604911 114, e-mail: an-a1@o2.pl

Received: 2.04 .2013 , accepted: 23.06 .2013$. 
ination disclosed precancerous keratosis. Treatment with fusidic acid caused improvement in a few weeks, but in place of the ulcer a new scar with tendency to hypertrophy developed. Additionally, purulent lesions were still present. Dermatological treatment was administered but purulent lesions recurred.

During psychiatric hospitalization the patient was consulted by a dermatologist who diagnosed self-inflicted skin lesions based on clinical presentation (presence of papules, erosions, crusts, and scars of bizarre shape (Figures 1, 2)). Lesions were sharply demarcated from healthy skin. Primary acne skin lesions like comedones were absent. The second histopathology taken from the thigh revealed: epidermis unchanged, perivascular lymphoid infiltrations with isolated neutrophils - suspicion of dermatitis artefacta. Dermatological treatment (occlusive dressing, tetracycline and hydrocortisone ointment, allantoin cream) was introduced with a complete improvement within 2 months. After antipsychotic treatment (olanzapine $20 \mathrm{mg} /$ daily) his mental state partially improved.

\section{Discussion}

Dermatitis artefacta is a disease that occurs as a result of a self-inflicted injury of the skin. It is characterized by a vast spectrum of geometrical or linear shape lesions such as: blisters, cuts, excoriations, ulcerations and purpura inflicted by means of various activities like: rubbing, sucking, biting, scratching, picking, cutting, slashing, gouging, puncturing, application of caustic or hot agents and injections of various substances [2-6]. Unlike other disorders of self-mutilation nature (e.g. neurotic excoriations, Munchausen syndrome, true malingering), creation of new lesions in dermatitis artefacta is usually involuntary $[5,6]$. Skin lesions are most often located on the areas within easy reach of the patient's dominant hand, usually sparing the middle part of the back. They are usu-

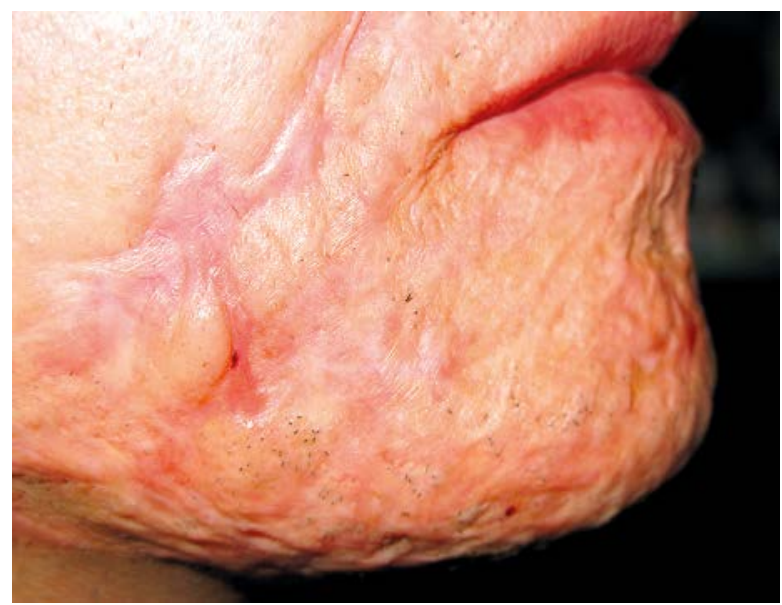

Figure 1. Hypertrophic scars on the chin and cheeks ally in different stage of healing and often demonstrate postinflammatory hyperpigmentation. Histopathological examination is generally non-specific [7]. Although our patient's lesions were considered to be both acne, bacterial lesions and tuberculosis, they presented features characteristic of dermatitis artefacta, namely they had mostly a geometrical shape and were located within easy reach of the patient's hands. The patient was not able to explain the history of their evolution, and although he acknowledged frequent touching of his skin, he still claimed that skin lesions resulted from: "whole sebum which goes out from my body" adding that: "my skin fissures, purulent lesions appear, little balls emerge from them".

Harth et al. noticed that dermatitis artefacta may coexist with psychiatric disorders [7]. It can be interpreted as a re-activation of a childhood injury or trauma such as sexual/physical abuse or neglect and represent a non-verbal "cry for help" $[2,8]$. Like in our case, the harmful behavior may be associated with psychotic disorders. It is known that major self-mutilation may be the main symptom of the first psychotic episode. Large et al. showed that $66.12 \%$ of patients with major self-mutilation were eventually diagnosed as schizophrenic psychosis [9]. Dermatitis artefacta can also coexist with personality disorders. Patients damage their skin to satisfy

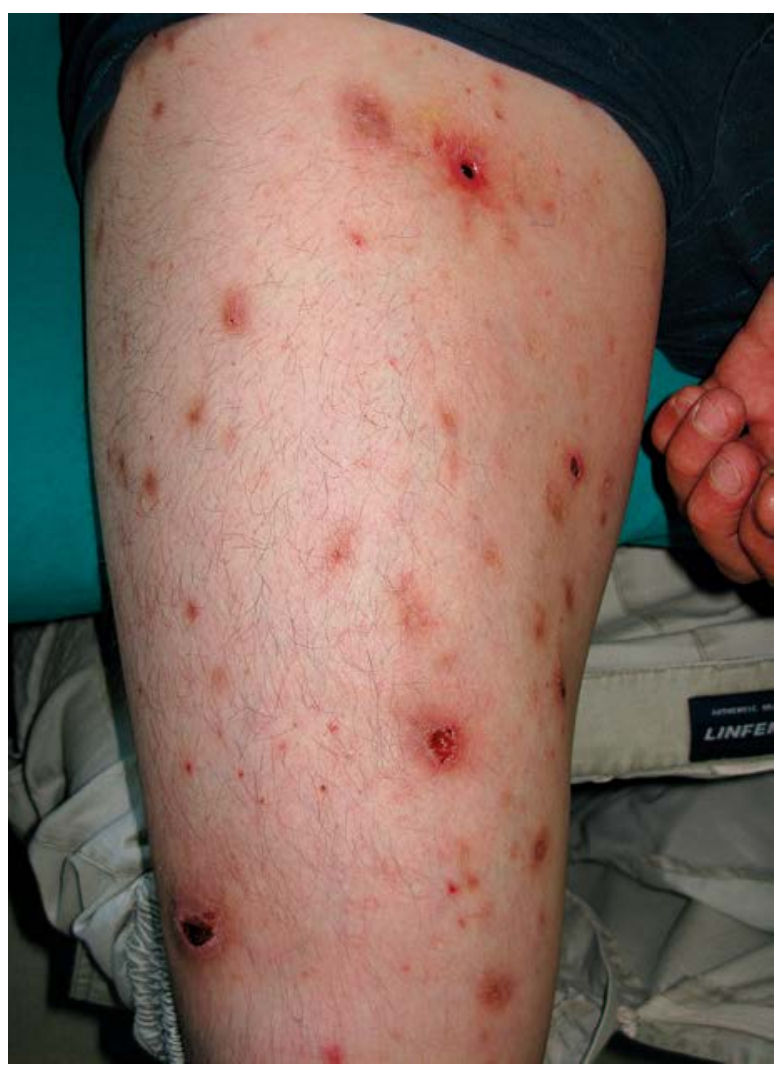

Figure 2. Papules, erosions, ulcers and crusts on the leg 
an unconscious need to be taken care of or to diminish their anxiety (because such behavior leads to release of endorphins) [10, 11].

As most of the patients with self-inflicted dermatoses usually initially deny any psychiatric problems, what delays psychiatric intervention, they are typically first seen by the dermatologist [11]. Our patient had been treated by a dermatologist for 7 years without psychiatric examination. Therefore, knowledge of these disorders is very important for both dermatologists and psychiatrists.

We suppose that infliction of skin lesions by our patient resulted from his psychotic symptoms (e.g. hypochondriac delusions, somatic hallucinations). Our conclusion seems to be confirmed by improvement of the skin condition after antipsychotic treatment.

\section{Conclusions}

As dermatitis artefacta may imitate many diseases, bearing in mind the self-inflicted nature of the lesions is always required, and it seems to be a huge challenge in numerous cases. Patients often consult different doctors for many years without being given a proper diagnosis. Consequently, they suspect that their doctors are incompetent, resulting in poor compliance and non-effective treatment. They often tend to discontinue therapy and the patient's medical history has significant gaps [8]. Such situation makes establishment of the proper diagnosis more and more difficult, finally leading to vicious circle formation. For our patient this process took 7 years (with 5-year break because of lack of improvement and high costs of dermatological treatment). During this time he was on many medicines e.g. isotretinoin, which is contraindicated in psychosis as it seems to worsen the course of the disease [12]. As the patient did not take any antipsychotic medicines, his delusional system gradually expanded and influenced his life causing work and marriage problems. Of great importance is also introducing treatment in early stage of the long-term course psychosis [13]. We would also like to draw attention to the fact that biopsies, microbiological examinations, blood tests, consultations, opinions, hospitalizations and drugs which are usually administered before stating the final diagnosis are very expensive [14]. We would like to recommend high awareness of self-inflicted skin lesions in both dermatologic and psychiatric patients and encourage dermatologists and psychiatrists to close cooperation.

\section{Acknowledgments}

The first and the second author equally contributed to the manuscript.

The work was financially supported by Medical University of Lodz (grant no. 503/1-137-04/503-01 and 50203/1-137-02/502-14-165).

\section{Conflict of interest}

There is no conflict of interest.

\section{References}

1. Tamakuwala B, Shah P, Dave K, Mehta R. Dermatitis artefacta. Indian J Psychiatry 2005; 47: 233-4.

2. Zalewska A. Clinical picture of self-inflicted skin lesions. Dermatol Psychosom 2004; 5: 79-84.

3. Rosińska A, Walkowiak H, Żaba R. Dermatitis artefacta - opis przypadku Postep Derm Alergol 2007; 14: 290-6.

4. Maio P, Santos R, Cardoso J. Letter: Factitial dermatitis: an unusual presentation in an old woman. Dermatol Online J 2012; 18: 10.

5. Bergler-Czop B, Brzezińska-Wcisło L. Psychodermatologic disorders: case reports and review of literature. Postep Derm Alergol 2012; 29: 401-6.

6. Joe E, Li V, Magro C, et al. Diagnostic clues to dermatitis artefacta. Cutis 1999; 63: 209-14.

7. Harth W, Taube KM, Gieler U. Facticious disorders in dermatology. J Dtsch Dermatol Ges 2010; 8: 361-72.

8. Large M, Babdge N, Andrews D, et al. Major self-mutilation in the first episode of psychosis. Schizophr Bull 2009; 35: 1012-21.

9. Millrd LG, Cotteril JA. Psychocutaneous disorders. In: Rook's textbook of dermatology. Burns T, Breathnach S, Cox N, et al. Blackwell Science, Oxford 2004; 61: 1-5.

10. Cotterill JA. Self-stigmatization: artefact dermatitis. $\mathrm{Br} J$ Hosp Med 1992; 47: 115-8.

11. Woodruff PWR, Higgins EM, Vivier AWP, Wessley S. Psychiatric illness in patients referred to dermatology-psychiatry clinic. Gen Hosp Psych 1997; 19: 29-35.

12. O'Donnell J. Overview of existing research and information linking isotretinoin (accutane), depression, psychosis, and suicide. Am J Ther 2003; 10: 148-59.

13. Schennach R, Riedel M, Musil R, Möller HJ. Treatment response in first-episode schizophrenia. Clin Psychopharmacol Neurosci 2012; 10: 78-87.

14. Barańska-Rybak W, Cubała WJ, Kozicka D, et al. Dermatitis artefacta - a long way from the first clinical symptoms to diagnosis. Psychiatr Danub 2011; 23: 73-5. 